\title{
Medical Therapy of Gastrointestinal Neuroendocrine Tumors
}

\author{
Kjell Öberg \\ Department of Endocrine Oncology, Uppsala University Hospital, Uppsala, Sweden
}

\section{Keywords}

Small intestinal neuroendocrine tumors .

Somatostatin analogs . Peptide receptor radionuclide therapy, PRRT · Targeted agents · Chemotherapy

\section{Summary}

Intestinal neuroendocrine tumors (NETs) constitute a heterogeneous group with duodenal, small intestinal, colonic and rectal NETs. They constitute more than half of all NETs, with the highest frequencies in the rectum, small intestine, and colon. The tumor biology varies with the location of the primary tumor as well as with the grade and staging of the tumor. Small intestinal NETs usually present low proliferation and are treated in the first line with somatostatin analogs according to current guidelines. If progression occurs, one can add interferon alpha or change the treatment to everolimus. Peptide receptor radionuclide therapy (PRRT) with Lutetium ${ }^{177}$-DOTATATE can be an option in the future after registration of the compound. Rectal tumors are usually small when they metastasize; they can be treated with somatostatin analogs but more so with PRRT, while another option is of course everolimus. Colonic NETs are more aggressive than the rest of intestinal NETs and will be treated with everolimus, sometimes in combination with somatostatin analogs based on positive scintigraphy. Another option is a cytotoxic agent such as streptozotocin plus 5-fluorouracil (5FU) or temozolomide plus capecitabine. The most aggressive tumors, i.e. neuroendocrine carcinoma G3, are treated with a platin-based therapy plus etoposide; if they present with a lower proliferation, i.e. $<50 \%$, temozolomide plus capecitabine plus bevacizumab can also be attempted. Duodenal NETs are mostly treated similar to pancreatic NETs, either with cytotoxic agents, streptozotocin plus 5-FU, or temozolomide plus capecitabine, or with targeted agents such as everolimus.

(c) 2017 S. Karger GmbH, Freiburg

\section{Introduction}

Intestinal neuroendocrine tumors (NETs) constitute a heterogeneous group with duodenal, small intestinal, colonic and rectal NETs. In the most recent SEER register (SEER-17), more than half of all NETs, i.e. $61 \%$, were gastroenteropancreatic neuroendocrine tumors (GEP-NETs), with the highest frequency being found in the rectum (17.7\%), the small intestine (17.3\%), and the colon (10.1\%) [1]. The tumor biology varies with the location of the primary tumors as well as with the grade and staging of the tumors. The malignant potential ranges from the most benign types of tumor to small intestinal tumors and up to colonic neuroendocrine carcinoma (NEC) with very malignant behavior [2]. The tumors are graded according to the classification system of the World Health Organization (WHO), whereas a new classification system is just being accepted. The tumors are divided into grade 1 NET (NET-G1), with a proliferation $<3 \%$, NET-G2 with a proliferation between 3 and 20\%, NET-G3, which is a new group with a Ki-67 $>20 \%$, and finally NEC-G3, exhibiting a Ki-67 of $>20 \%$ as well (unpublished data). Of note, the difference between NET-G3 and NEC-G3 is mainly the degree of differentiation. NET-G3 are well-differentiated tumors, often with expression of somatostatin receptors. NEC-G3 are poorly differentiated tumors that usually lack expression of somatostatin receptors. Furthermore, the tumors are classified according to the staging systems ENETS and UICC (Union for International Cancer Control)/ AJCC (American Joint Committee on Cancer) depending on the spread of the disease [3-5]. The genetic background information on intestinal NETs is still limited. It is well known that small intestinal NETs display chromosomal losses on chr 18 and cyclindependent kinase inhibitor mutations $[5,6]$. However, genetic changes, such as mutations, are very rare in this group of tumors. When they become more malignant, such as NEC-G3, more genetic changes are found, e.g. Rb and TP 53 mutations [7, 8]. Epigenetic changes (DNA methylation) are noticed in methyl guanine methyl transferase (MGMT), ras association domain family

\section{KARGER}

() 2017 S. Karger GmbH, Freiburg

Fax +497614520714 
1A (RASSF1A), and cyclin-dependent kinase inhibitor 2A (CDKN2A) $[9,10]$. Intestinal NETs express a number of G-proteincoupled receptors where the somatostatin receptors are the most frequent and target for correct staging of the disease, evaluation of therapeutic responses, and treatment $[11,12]$.

\section{Small Intestinal Tumors (SI-NETs Ileum, Jejunum)}

\section{Somatostatin Analogs}

The medical treatment is very important for small intestinal NETs (SI-NETs) since more than $60 \%$ of the patients present with metastatic disease at diagnosis $[13,14]$. The first-line treatment for SI-NETs is somatostatin analogs, both to control clinical symptoms related to hormone hypersecretion and to control the tumor growth [15-20]. Short-acting octreotide (developed in 1982) was the first agent used for the successful control of symptoms associated with carcinoid tumors [21]. Later on, a long-acting formulation of octreotide was developed, i.e. octreotide LAR, showing similar efficacy as the short-acting formulation in the treatment of carcinoid syndrome. Today, octreotide LAR has replaced the treatment with short-acting octreotide $[17,22]$. Sometimes short-acting forms might be added to the long-acting formulation to control clinical symptoms such as flushing and diarrhea. Old data from studies of octreotide in GEP-NETs conducted between 1986 and 2004 indicated that up to $70 \%$ of the patients experience resolution of diarrhea or flushing with octreotide treatment [16]. Lanreotide, another somatostatin analog, has a similar efficacy as octreotide in reducing flushes and diarrhea. Patients with carcinoid syndrome achieve a relief of symptoms in up to $80 \%$. Today, lanreotide only exists as a long-term formulation (Somatuline autogel) [23]. With the introduction of somatostatin analogs, there was a growing body of evidence that they might also improve survival in patients with SI-NETs as well as other slow-growing GEP-NETs $[15,16]$. The antitumor effect of octreotide in clinical studies of patients indicated that with the use of somatostatin analogs in SI-NETs, stabilization of tumor growth occurred in about $50 \%$ of the patients while only $10 \%$ showed tumor regression. A recent review that analyzed trials conducted between 1987 and 2011 revealed that stable disease in patients with well-differentiated functioning and non-functioning GEP-NETs was achieved in up to $86 \%$ when treated with octreotide subcutaneous or LAR [24]. A tumor reduction was noticed in $11 \%$ of the patients. For lanreotide autogel, a study with well-differentiated GEP-NETs showed stable disease and partial tumor response in 89 and $4 \%$ of the patients, respectively [23]. PROMID was the first large trial to confirm the antitumor effect of octreotide LAR in a randomized setting in 95 treatment-naive patients with well-differentiated SI-NETs. The medium time to tumor progression was significantly extended, i.e. 14.3 months for octreotide LAR (30 mg/month) compared with 6 months for placebo. Stable disease was observed in 67 or $37 \%$ of the patients treated with octreotide LAR or placebo, respectively. The antitumor response was more pronounced in patients with a hepatic tumor burden of $<10 \%$. No difference in response was reported between functioning and non-functioning SI-NETs [18]. The antiproliferative effect of lanreotide autogel in patients with GEPNETs was evaluated in the so-called CLARINET study, which is a phase III randomized study, confirming the antitumor efficacy of somatostatin analogs. 204 medically naïve patients with welldifferentiated or moderately differentiated non-functioning tumors were treated with autogel $120 \mathrm{mg}$ or placebo every 4 weeks. Medium progression-free survival (PFS) was not reached with lanreotide autogel after 2 years versus 18 months with placebo. After 2 years of treatment, the estimated rates of PFS were 65.1 and $33 \%$ in the lanreotide autogel and placebo groups, respectively [19]. There was a major difference between the PROMID and the CLARINET study accounting for the longer PFS observed in the latter study. The population in the CLARINET study was more heterogeneous, including not only SI-NETs but also pancreatic NETs with a Ki-67 value of $<10 \%$. The patients also had a clinically stable disease when starting the study. In the PROMID study, some patients showed progressing disease while some had stable disease. With almost three decades of clinical experience with somatostatin analogs, a well-established safety profile can be noted. Gastrointestinal complaints are the most frequent side effects; these are light to moderate in severity, can be attributed to drug-induced disruption of GEP hormone signaling, and reduce the secretion of digestive enzymes. Altered secretion of cholecystokinin can lead to abnormalities in the biliary system, and development of sludge and gallstones has been reported in about half of the patients [25]. Other side effects, which are rather rare, are bradycardia and impaired glucose tolerance. Pasireotide LAR, another somatostatin analog which binds to 4 out of 5 somatostatin receptors, has demonstrated symptom control in patients with SI-NETs in a phase II multicenter study in 44 patients with advanced SI-NETs whose symptoms were refractory or resistant to regular doses of octreotide LAR up to 30 mg. Pasireotide treatment provides relief of symptoms such as diarrhea and flushing in $27 \%$ of the patients [26]. Additionally, in a phase III study of pasireotide LAR versus high-dose $(40 \mathrm{mg})$ octreotide LAR in 110 patients with advanced GEP-NETs whose disease-related symptoms were uncontrolled by first-generation somatostatin analogs at maximum approved doses, pasireotide LAR and octreotide had a similar effect on symptom control [27]. Further development of pasireotide LAR in GEP-NETs is on hold, and the precise role of this somatostatin analog in the future treatment of SI-NETs is uncertain. The incorporation of somatostatin analogs into radiopharmaceuticals allows for targeted delivery of radiation to somatostatin receptor-expressing tumors. The efficacy of $\mathrm{Lu}^{177}$-DOTATATE, a Lutathera ${ }^{\circledR}$ conjugate consisting of octreotate radiolabeled with $\mathrm{Lu}^{177}$, has been evaluated in patients with advanced SI-NETs in the NETTER-1 phase III study. 229 patients were randomized to receive $\mathrm{Lu}^{177}$ DOTATATE or combined with octreotide LAR $30 \mathrm{mg}$. There were significant differences in both PFS and response rate as well as in the overall survival (in the interim analysis) in favor of $\mathrm{Lu}^{177}$-DOTATATE [28]. When $\mathrm{Lu}^{177}$-DOTATATE will be ap- 
proved for SI-NETs by the European Medicines Agency (EMA) and the Food and Drug Administration (FDA), it might be considered as a second-line therapy.

\section{Interferon Alpha}

Interferon alpha is a second-line therapy in NETs that are functionally active with low proliferation capacity, such as SI-NETs and well-differentiated pancreatic NETs with low proliferation [29, 30]. It is recommended to use interferon alpha as add-on therapy to somatostatin analogs in functional tumors. The recommended dose of subcutaneous interferon alpha- $2 \mathrm{~b}$ is $3 \times 3$ to $3 \times 5$ million units per week. In patients that do not tolerate the conventional regimen, alternative pegylated interferon alpha (50-180 $\mu \mathrm{g}$ /week subcutaneous) can also be used [31]. Interferon alpha has an antiproliferative activity and may be considered for antiproliferative purposes if other approved drugs are unavailable, especially in SI-NETs [30]. Interferon alpha has been explored in comparison with bevacizumab for antiproliferative purposes in a large randomized trial of 400 patients with carcinoids (including different primary sites) who received octreotide LAR concomitantly (SWOG trial). The medium PFS as the primary endpoint was not different between those taking interferon alpha and those taking bevacizumab. This study confirms the antiproliferative activity of interferon alpha- $2 b$ in advanced gastrointestinal NET-G2 with progressive disease or with other poor prognostic features and with a medium PFS of 15.4 months reached in the interferon alpha arm [32].

\section{Novel Targeted Drugs}

Everolimus can be recommended in advanced SI-NETs based on results from the RADIANT-4 trial, which is indicating that everolimus compared to placebo in non-functioning SI-NETs showed a superior PFS. This is further supported by data from the RADIANT-2 trial on advanced NETs associated with a carcinoid syndrome $[33,34]$. The sequencing of everolimus as second- or third-line treatment for advanced intestinal NETs depends on other issues including the accessibility of PRRT. Individual patient selection is important, and it is a standard practice to combine targeted drugs with somatostatin analogs in functionally active neuroendocrine neoplasms (NENs). The aim of a combination therapy of everolimus and somatostatin analogs may not only be the inhibition of tumor growth but also improved syndrome control. A treatment algorithm is suggested in the ENETS guidelines [20].

\section{Other Therapy}

Recently, the new compound telotristat ethyl, which is a tryptophan hydroxylase inhibitor that reduces the circulating levels of serotonin, has been introduced. In the Telstar and Telecast trials, patients with carcinoid syndrome and specific problems with diarrhea on recommended doses of somatostatin analogs received two doses of telotristat ethyl, i.e. 250 and $500 \mathrm{mg}$, versus placebo. There was a significant reduction in bowel movements but daily flushes remained unchanged; urinary 5-hydroxyindoleacetic acid levels as well as abdominal pain were significantly reduced [35]. The drug has now been registered in the USA for the treatment of patients with carcinoid syndrome. It will be interesting to see in the future if a reduction of serotonin will prevent the development of carcinoid heart disease as well.

\section{Concluding Remarks}

Somatostatin analogs are considered to be the first-line treatment in SI-NETs with low proliferation, usually less than $2 \% \mathrm{Ki}-$ 67. It can be combined with alpha interferon in patients that are resistant to somatostatin analogs. As a second-line treatment, data from the NETTER-1 study is supporting the use of PRRT in this group of patients; in patients which are lacking somatostatin receptor expression, however, everolimus might be an alternative. Combined therapies of somatostatin analogs and targeted agents such as everolimus are recommended in patients with more aggressive tumors as well as in those with a higher tumor burden.

\section{Colorectal Neuroendocrine Tumors and Neuroendocrine Carcinoma}

Rectal NETs occur more often than SI-NETs [1], and the incidence is higher in patients from the Asia-Pacific region. It is important to realize that rectal NETs differ from colonic NETs. Rectal NETs are usually small $(<2 \mathrm{~cm})$ with localized disease and in general low to intermediate grade 1 or $2(\mathrm{G} 1, \mathrm{G} 2)$. The 5 -year survival rate is about $90 \%$. In contrast, colonic NETs are often aggressive as well as poorly differentiated with higher grade (G3). Most NETs of the colon arise in the cecum and ascending colon. The overall survival (5 years) is $40-70 \%$ depending on the specific site and stage [36]. Poorly differentiated NECs (large and small cell) present 5 -year survival rates similar to adenocarcinomas [37, 38]. The medical treatment of metastatic rectal NETs consists of a combination of everolimus and octreotide according to some data from the RADIANT-2 trial. It seems somewhat irrational to be using this combination in well-differentiated G1 and G2 rectal NETs but it remains to be verified [34]. Somatostatin analogs alone have also been applied. There is a small group of patients to be found in the CLARINET study; however, 14 cases of colorectal NETs are not enough to conduct an evaluation of the real benefit [19]. Nevertheless, many of the rectal NETs (G1 and G2) express a lot of somatostatin receptors (type 2). Therefore, radioactive treatment with $\mathrm{Lu}^{177}$-DOTATATE looks very promising, although no randomized control trial has been conducted so far. NEC-G3 constitute about $5 \%$ of all gastrointestinal tumors and have a Ki-67 $>20 \%$ [37]. The highest frequency of NECs is in the colon, with 40 and $25 \%$ of the cases having metastases at the time of diagnosis, thus not being amenable to surgical cure [39]. Chemotherapy is the mainstay of care in metastatic disease. First-line therapy usually consists of cisplatin or carboplatin combined with etoposide. Other combinations of chemotherapy have been irinotecan/cisplatin and oxaliplatin-based therapies (XELOX/FOLFOX) or irinotecan-based FOLFIRI regimens [40, 41]. Temozolomide alone or in combination with capecitabine or bevacizumab has been effective as second- or third-line treatment of NECs with a Ki-67 of $<50 \%$ [42]. 


\section{Concluding Remarks}

Rectal NETs are usually small well-differentiated tumors which can be treated locally. Metastatic tumors can be treated with everolimus or PRRT. Colonic NETs/NECs are usually treated with chemotherapy or targeted agents.

\section{Gastroduodenal Neuroendocrine Tumors}

Gastric NETs represent the most frequent digestive NETs and are increasingly detected due to expanding indications of upper gastrointestinal endoscopy [43, 44]. Often silent and benign, gastric NETs may still be aggressive and sporadic and may sometimes mimic the course of gastric adenocarcinoma [45]. Duodenal NETs may be sporadic or associated with multiple neuroendocrine neoplasia type 1 (MEN-1) and present with a functional syndrome such as Zollinger-Ellison syndrome (ZES). Other duodenal NETs might secrete somatostatin and be part of von Hippel-Lindau disease [46, 47]. New data indicate that gastric NETs and duodenal NETs represent 6.9 and $2 \%$ of all digestive NETs, respectively [43]. Well-differentiated gastric NETs may be divided into three types, whereas type 1 and type 2 are ECL-omas (enterochromaffin cell-like tumors) due to chronic hypergastrinemia associated with chronic gastritis, atrophic gastritis, or ZES. Type 3 gastric NETs are rare, sporadic, and not a consequence of an underlying gastric mucosal abnormality. They are mostly single, large lesions with high-grade metastatic potential (G3-NEC) [48-50]. The overall outcome in type 1 gastrointestinal NENs is excellent with a survival rate of almost $100 \%$. The medical treatment of patients with type 1 gastric NETs includes therapy with somatostatin analogs and has been used in limited series which led to a regression of the tumor. Somatostatin analogs might be useful to treat patients with multiple small lesions that are hard to eradicate endoscopically [51]. The gastrin receptor antagonist netazepide has been shown to have antiproliferative properties in gastric NETs in non-controlled studies [52,53]. For type 2 gastric NETs, treatment with both somatostatin analogs and netazepide is possible; yet again, randomized controlled studies are not available. In patients with type 3 gastric NETs, the disease is spread, whereas in stage 4 disease, systemic therapy is indicated based on differentiation. Systemic therapy in line with other gastrointestinal malignancies is recommended. For duodenal tumors, if metastatic and locally advanced, treatment with targeted agents such as everolimus can be attempted; otherwise, cytotoxic treatment with temozolomide, streptozotocin alone or in combination with 5-FU, or capecitabine are other options. Furthermore, PRRT with $\mathrm{Lu}^{177}$-DOTATATE can be applied, provided that the tumor has a significant expression of somatostatin receptors. No randomized controlled trials exist for this group of patients.

\section{Concluding Remarks}

Type 1 and 2 gastric NETs can be treated with somatostatin analogs or the gastrin receptor antagonist netazepide. For metastatic duodenal NETs and also gastric NEN-G3, systemic chemotherapy or targeted agents might be applied. However, limited data are still available regarding the value of these therapies. Radioactive treatment with PRRT can be applied in gastroduodenal NETs with a high expression of somatostatin receptors [53].

\section{Conclusion}

Gastrointestinal NETs are a heterogeneous group of neoplasms with varying clinical and biological features. The treatment must be individualized for the subgroups of tumors as well as for the patients.

\section{Glossary}

- SEER: Surveillance, epidemiology, and end results.

- PROMID: Placebo-controlled, double-blind, prospective, randomized study on the effect of octreotide LAR in the control of tumor growth in patients with metastatic neuroendocrine midgut tumors.

- CLARINET: Lanreotide antiproliferative response in patients with GEPNETs.

- SWOG: A National Cancer Institute(NCI)-supported organization that conducts clinical trials in adult cancers (formerly the Southwest Oncology Group).

- RADIANT-2: A randomized, double-blind placebo-controlled, multicenter phase III study in patients with advanced carcinoid tumor receiving octreotide depot and everolimus $10 \mathrm{mg} /$ day or octreotide depot and placebo.

\section{Disclosure Statement}

Novartis: Grant and honoraria. IPSEN: Honoraria.

\section{References}

1 Lawrence B, Gustafsson BI, Chan A, Svejda B, Kidd M, Modlin IM: The epidemiology of gastroenteropancreatic neuroendocrine tumors. Endocrinol Metab Clin North Am 2011;40:1-18, vii.

2 Tang LH, Untch BR, Reidy DL, O’Reilly E, Dhall D, Jih L, Basturk O, Allen PJ, Klimstra DS: Well-differentiated neuroendocrine tumors with a morphologically apparent high-grade component: a pathway distinct from poorly differentiated neuroendocrine carcinomas. Clin Cancer Res 2016;22:1011-1017.
3 Klöppel G, Rindi G, Perren A, Komminoth P, Klimstra DS: The ENETS and AJCC/UICC TNM classifications of the neuroendocrine tumors of the gastrointestinal tract and the pancreas: a statement. Virchows Arch 2010;456:595-597.

4 Klöppel G: Classification and pathology of gastroenteropancreatic neuroendocrine neoplasms. Endocr Relat Cancer 2011;18(suppl 1):S1-16.

5 Banck MS, Kanwar R, Kulkarni AA, et al: The genomic landscape of small intestine neuroendocrine tumors. J Clin Invest 2013;123:2502-2508.
6 Francis JM, Kiezun A, Ramos AH, et al: Somatic mutation of CDKN1B in small intestine neuroendocrine tumors. Nat Genet 2013;45:1483-1486.

7 Tang LH, Contractor T, Clausen R, Klimstra DS, Du YC, Allen PJ, Brennan MF, Levine AJ, Harris CR: Attenuation of the retinoblastoma pathway in pancreatic neuroendocrine tumors due to increased cdk4/cdk6. Clin Cancer Res 2012;18:4612-4620. 
8 La Rosa S, Marando A, Furlan D, Sahnane N, Capella C: Colorectal poorly differentiated neuroendocrine carcinomas and mixed adenoneuroendocrine carcinomas: insights into the diagnostic immunophenotype, assessment of methylation profile, and search for prognostic markers. Am J Surg Pathol 2012;36:601-611.

9 Stricker I, Tzivras D, Nambiar S, Wulf J, Liffers ST, Vogt M, Verdoodt B, Tannapfel A, Mirmohammadsadegh A: Site- and grade-specific diversity of LINE1 methylation pattern in gastroenteropancreatic neuroendocrine tumours. Anticancer Res 2012;32:3699-3706

10 Dammann R, Schagdarsurengin U, Strunnikova M, Rastetter M, Seidel C, Liu L, Tommasi S, Pfeifer GP: Epigenetic inactivation of the Ras-association domain family 1 (RASSF1A) gene and its function in human carcinogenesis. Histol Histopathol 2003;18:665-677.

11 Reubi JC: Peptide receptors as molecular targets for cancer diagnosis and therapy. Endocr Rev 2003;24: 389-427.

12 Reubi JC, Waser B: Concomitant expression of several peptide receptors in neuroendocrine tumours: molecular basis for in vivo multireceptor tumour targeting. Eur J Nucl Med Mol Imaging 2003;30:781-793.

13 Strosberg J: Neuroendocrine tumours of the small intestine. Best Pract Res Clin Gastroenterol 2012;26: 755-773.

14 Ahmed A, Turner G, King B, et al: Midgut neuroendocrine tumours with liver metastases: results of the UKINETS study. Endocr Relat Cancer 2009;16:885894.

15 Öberg K, Kvols L, Caplin M, Delle Fave G, De Herder W, Rindi G, Ruszniewski P, Woltering EA, Wiedenmann B: Consensus report on the use of somatostatin analogs for the management of neuroendocrine tumors of the gastroenteropancreatic system. Ann Oncol 2004; 15:966-973.

16 Eriksson B, Öberg K: Summing up 15 years of somatostatin analog therapy in neuroendocrine tumors: future outlook. Ann Oncol 1999;10(suppl 2):S31-38.

17 Kvols LK, Moertel CG, O'Connell MJ, Schutt AJ, Rubin J, Hahn RG: Treatment of the malignant carcinoid syndrome. Evaluation of a long-acting somatostatin analogue. N Engl J Med 1986;315:663-666.

18 Rinke A, Muller HH, Schade-Brittinger C, et al: Placebo-controlled, double-blind, prospective, randomized study on the effect of octreotide LAR in the control of tumor growth in patients with metastatic neuroendocrine midgut tumors: a report from the PROMID Study Group. J Clin Oncol 2009;27:4656-4663.

19 Caplin ME, Pavel M, Cwikla JB, et al.; CLARINET Investigators: Lanreotide in metastatic enteropancreatic neuroendocrine tumors. N Engl J Med 2014;371 224-233.

20 Pavel M, O’Toole D, Costa F, et al: ENETS Consensus Guidelines update for the management of distant metastatic disease of intestinal, pancreatic, bronchial neuroendocrine neoplasms (NEN) and NEN of unknown primary site. Neuroendocrinology 2016;103:172-185.

21 Bauer W, Briner U, Doepfner W, Haller R, Huguenin R, Marbach P, Petcher TJ, Pless: SMS 201-995: a very potent and selective octapeptide analogue of somatostatin with prolonged action. Life Sci 1982;31:1133-1140.

22 Lancranjan I, Bruns C, Grass P, et al: Sandostatin LAR: a promising therapeutic tool in the management of acromegalic patients. Metabolism 1996;45(suppl 1):67-71.

23 Martín-Richard M, Massuté B, Pineda E, et al: Antiproliferative effects of lanreotide autogel in patients with progressive, well-differentiated neuroendocrine tumours: a Spanish, multicentre, open-label, single arm phase II study. BMC Cancer 2013;13:427.
24 Sideris L, Dube P, Rinke A: Antitumor effects of somatostatin analogs in neuroendocrine tumors. Oncologist 2012;17:747-755

25 Trendle MC, Moertel CG, Kvols LK: Incidence and morbidity of cholelithiasis in patients receiving chronic octreotide for metastatic carcinoid and malignant islet cell tumors. Cancer 1997;79:830-834.

26 Kvols LK, Öberg KE, O’Dorisio TM, et al: Pasireotide (SOM230) shows efficacy and tolerability in the treatment of patients with advanced neuroendocrine tumors refractory or resistant to octreotide LAR: results from a phase II study. Endocr Relat Cancer 2012;19: 657-666.

27 Wolin EM, Jarzab B, Eriksson B, et al: Phase III study of pasireotide long-acting release in patients with metastatic neuroendocrine tumors and carcinoid symptoms refractory to available somatostatin analogues. Drug Des Devel Ther 2015;9:5075-5086.

28 Strosberg J, El-Haddad G, Wolin E, et al: Phase 3 trial of ${ }^{177} \mathrm{Lu}$-Dotatate for midgut neuroendocrine tumors. N Engl J Med 2017;376:125-135.

29 Eriksson B, Klöppel G, Krenning E, et al: Consensus guidelines for the management of patients with digestive neuroendocrine tumors - well-differentiated jejunal-ileal tumor/carcinoma. Neuroendocrinology 2008; 87:8-19.

30 Öberg K: Interferon-alpha versus somatostatin or the combination of both in gastro-enteropancreatic tumours. Digestion 1996;57(suppl 1):81-83.

31 Pavel ME, Baum U, Hahn EG, Schuppan D, Lohmann T: Efficacy and tolerability of pegylated IFN-alpha in patients with neuroendocrine gastroenteropancreatic carcinomas. J Interferon Cytokine Res 2006;26:8-13.

32 Yao JC, Guthrie KA, Moran C, et al: Phase III prospective randomized comparison trial of depot octreotide plus interferon alfa-2b versus depot octreotide plus bevacizumab in patients with advanced carcinoid tumors: SWOG S0518. J Clin Oncol 2017;35:1695-1703.

33 Yao JC, Fazio N, Singh S, et al: Everolimus for the treatment of advanced, non-functional neuroendocrine tumours of the lung or gastrointestinal tract (RADIANT-4): a randomised, placebo-controlled, phase 3 study. Lancet 2016;387:968-977.

34 Pavel ME, Hainsworth JD, Baudin E, et al: Everolimus plus octreotide long-acting repeatable for the treatment of advanced neuroendocrine tumours associated with carcinoid syndrome (RADIANT-2): a randomised, placebo-controlled, phase 3 study. Lancet 2011;378:2005-2012.

35 Lamarca A, Barriuso J, McNamara MG, Hubner RA, Valle JW: Telotristat ethyl: a new option for the management of carcinoid syndrome. Expert Opin Pharmacother 2016;17:2487-2498.

36 Modlin IM, Öberg K, Chung DC, et al: Gastroenteropancreatic neuroendocrine tumours. Lancet Oncol 2008;9:61-72.

37 Mandair D, Caplin ME: Colonic and rectal NET's. Best Pract Res 2012;26:775-789.

38 Caplin M, Sundin A, Nillson O, Baum RP, Klose KJ, Kelestimur F, Plockinger U, Papotti M, Salazar R, Pascher A: ENETS Consensus Guidelines for the management of patients with digestive neuroendocrine neoplasms: colorectal neuroendocrine neoplasms. Neuroendocrinology 2012;95:88-97.

39 Niederle MB, Hackl M, Kaserer K, Niederle B: Gastroenteropancreatic neuroendocrine tumours: the current incidence and staging based on the WHO and European Neuroendocrine Tumour Society classification: an analysis based on prospectively collected parameters. Endocr Relat Cancer 2010;17:909-918.
40 Sorbye H, Welin S, Langer SW, et al: Predictive and prognostic factors for treatment and survival in 305 patients with advanced gastrointestinal neuroendocrine carcinoma (WHO G3): the NORDIC NEC study. Ann Oncol 2013;24:152-160.

41 Yamaguchi T, Machida N, Morizane C, et al: Multicenter retrospective analysis of systemic chemotherapy for advanced neuroendocrine carcinoma of the digestive system. Cancer Sci 2014;105:1176-1181.

42 Welin S, Sorbye H, Sebjornsen S, Knappskog S, Busch C, Öberg K: Clinical effect of temozolomide-based chemotherapy in poorly differentiated endocrine carcinoma after progression on first-line chemotherapy. Cancer 2011;117:4617-4622.

43 O'Connor JM, Marmissolle F, Bestani C, et al: Observational study of patients with gastroenteropancreatic and bronchial neuroendocrine tumors in Argentina: results from the large database of a multidisciplinary group clinical multicenter study. Mol Clin Oncol 2014; 2:673-684.

44 Yao JC, Hassan M, Phan A, et al: One hundred years after 'carcinoid': epidemiology of and prognostic factors for neuroendocrine tumors in 35,825 cases in the United States. J Clin Oncol 2008;26:3063-3072.

45 Modlin IM, Lye KD, Kidd M: A 50-year analysis of 562 gastric carcinoids: small tumor or larger problem? Am J Gastroenterol 2004;99:23-32.

46 Mullen JT, Wang H, Yao JC, Lee JH, Perrier ND, Pisters PW, Lee JE, Evans DB: Carcinoid tumors of the duodenum. Surgery 2005;138:971-977; discussion 977-978.

47 Kölby L, Nilsson O, Ahlman H: Gastroduodenal endocrine tumours. Scand J Surg 2004;93:317-323.

48 Rindi G, Bordi C, Rappel S, La Rosa S, Stolte M, Solcia E: Gastric carcinoids and neuroendocrine carcinomas: pathogenesis, pathology, and behavior. World J Surg 1996;20:168-172.

49 La Rosa S, Inzani F, Vanoli A, Klersy C, Dainese L, Rindi G, Capella C, Bordi C, Solcia E: Histologic characterization and improved prognostic evaluation of 209 gastric neuroendocrine neoplasms. Hum Pathol 2011;42:1373-1384.

50 Rindi G, Azzoni C, La Rosa S, Klersy C, Paolotti D, Rappel S, Stolte M, Capella C, Bordi C, Solcia E: ECL cell tumor and poorly differentiated endocrine carcinoma of the stomach: prognostic evaluation by pathological analysis. Gastroenterology 1999;116:532-542.

51 Jianu CS, Fossmark R, Syversen U, Hauso O, Fykse V, Waldum HL: Five-year follow-up of patients treated for 1 year with octreotide long-acting release for enterochromaffin-like cell carcinoids. Scand J Gastroenterol 2011;46:456-463.

52 Fossmark R, Sordal O, Jianu CS, Qvigstad G, Nordrum IS, Boyce M, Waldum HL: Treatment of gastric carcinoids type 1 with the gastrin receptor antagonist netazepide (YF476) results in regression of tumours and normalisation of serum chromogranin A. Aliment Pharmacol Ther 2012;36:1067-1075.

53 Moore AR, Boyce M, Steele IA, Campbell F, Varro A, Pritchard DM: Netazepide, a gastrin receptor antagonist, normalises tumour biomarkers and causes regression of type 1 gastric neuroendocrine tumours in a nonrandomised trial of patients with chronic atrophic gastritis. PloS One 2013;8:e76462. 